\title{
Hacia la esencia de la arquitectura: el papel de Oriente en los años experimentales de Miguel Fisac
}

\author{
Óscar LORENTE ALCOYA* \\ Towards the essence of architecture: the role of the Orient in \\ Miguel Fisac's experimental years
}

\begin{abstract}
RESUMEN
Tanto la arquitectura tradicional japonesa como los pensamientos del filósofo chino Lao zi son referencias fundamentales en la obra de Miguel Fisac, uno de los arquitectos españoles más destacados de la segunda mitad del siglo XX. A pesar de haber reconocido estas influencias en numerosas ocasiones, este hecho todavía no ha sido objeto de un estudio profundo.

Sin embargo, el análisis de su obra y de su interés por Japón, a donde viajó varias veces, pone de relieve que la arquitectura española fue permeable a este influjo. El presente artículo aporta una visión más completa de la evolución de Fisac y ofrece

un panorama histórico de su desarrollo durante las décadas de los años cuarenta y cincuenta, cuando concibió su personal concepto de la arquitectura tomando ideas de diferentes fuentes, entre las que Oriente se encuentra en un lugar principal. Además, se examina en detalle la repercusión que el contacto con la arquitectura japonesa tuvo en su producción.
\end{abstract}

PALABRAS CLAVE

Arquitectura española; Movimiento Moderno; Oriente; Japón; Miguel Fisac.

\begin{abstract}
Japanese architecture as well as the thoughts of the Chinese philosopher Lao zi are fundamental references in the work of Miguel Fisac, one of the most renowned Spanish architects in the second half of the twentieth century. Despite having acknowledged said influences on many occasions, this matter has not yet been comprehensively studied. However, analysis of his work and his interest in Japan, where he travelled several times, demonstrate that the Spanish architecture was influenced by the Orient. This article provides a more complete overview of Fisac's evolution as an architect. It offers a historic outlook of his development during the forties and fifties, when he created his personal concept of architecture by appropriating ideas from diverse sources, with oriental architecture being one of his principal influences. In addition, this article examines, in detail, the effects that contact with Japanese architecture had on Fisac's output.
\end{abstract}

\section{KEYWORDS}

Spanish architecture; Modernism; Orient; Japan; Miguel Fisac.

* Arquitecto por la Universidad de Navarra, Máster en Estudios Avanzados en Historia del Arte de la Universidad de Zaragoza. Contacto: olorente@ coaaragon.org 


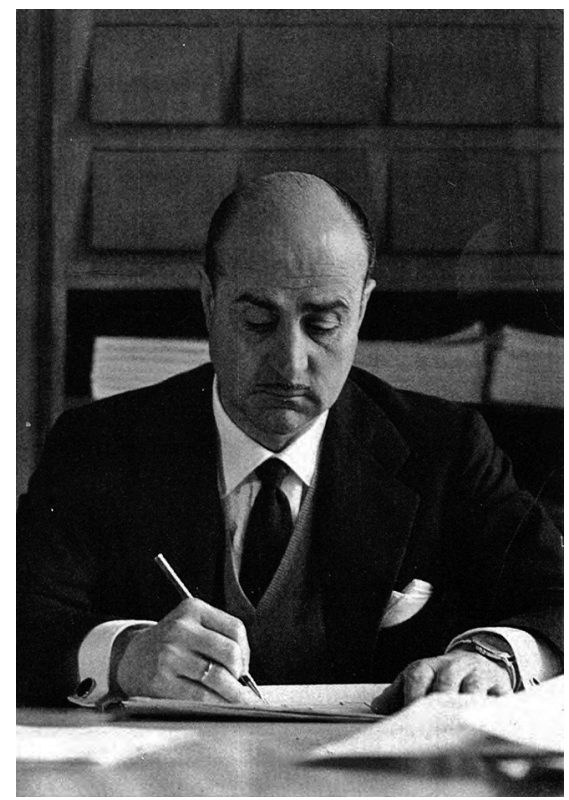

Fig. 1. Miguel Fisac en su estudio de Madrid, c. 1950.

\section{INTRODUCCIÓN}

Miguel Fisac Serna (Daimiel, 1913-Madrid, 2006) ha sido uno de los arquitectos españoles más importantes y reconocidos tanto en España como en el extranjero durante la segunda mitad del siglo XX (fig. 1).

Su obra tiene un carácter muy personal y se caracteriza por la experimentación. Por una parte, sus investigaciones arquitectónicas le condujeron a proponer numerosos avances técnicos (patentes de ladrillo y de hormigón), que se plasman en una obra profundamente expresiva, a la vez que muy rigurosa en su construcción. Por otra parte, llevó a cabo una notable experimentación espacial, que se aprecia, por ejemplo, en las numerosas iglesias que construyó. Esto supuso una radical renovación del espacio sagrado, que se adelantó a las directrices del Concilio Vaticano II (1962-1965).

Además, Fisac fue un incansable viajero y pudo evaluar de primera mano tanto la obra de los grandes maestros de la arquitectura moderna como la construcción en otros países; entre ellos, Japón, donde pudo observar el diferente modo de vida de esa nación e imbuirse de su refinado gusto estético, ya que la arquitectura y la jardinería japonesas le produjeron un gran impresión.

En este contexto se inscribe el presente artículo, en el que se ofrece un panorama histórico de la evolución arquitectónica de Fisac durante los años en los que 
desarrolló su personal concepto de la arquitectura. Es decir, en «los años experimentales», siguiendo la denominación que Fullaondo, en su análisis de la obra del arquitecto manchego, acuñó para referirse al periodo que abarca desde 1942 a 1959. En él, la producción de Fisac está condicionada por diversas corrientes e influencias, entre las que se encuentra la arquitectura japonesa como uno de sus "hallazgos» ${ }^{1}$. Sin embargo, desde ese momento, aunque las referencias orientales en su obra han sido planteadas en algunas ocasiones ${ }^{2}$ (e incluso fueron reconocidas por el propio Fisac), este es un tema que no se ha desarrollado suficientemente y que todavía no ha sido objeto de un estudio exhaustivo.

\section{LA ARQUITECTURA ESPAÑOLA DE POSGUERRA}

Miguel Fisac comienza sus estudios en la Escuela de Arquitectura de Madrid en 1932 y los finaliza en 1942, tras el paréntesis de la Guerra Civil (1936-1939), en la que no hubo docencia. Así, inicia su carrera profesional en plena posguerra, siendo uno de los pocos arquitectos — su promoción contaba con tan solo diez estudiantes- que en esos años se incorporaban al oficio.

Esta generación de arquitectos (Alejandro de la Sota, José Luis Fernández del Amo, Francisco de Asís Cabrero, Rafael Aburto, etc.) desarrolló su labor en una época marcada por unas duras condiciones tanto económicas como sociales y de manera casi «autodidacta» ${ }^{3}$. Por una parte, España se encontraba aislada de Europa, la cual estaba sumida en su propia guerra; por otra, la mayoría de los grandes arquitectos de la época anterior habían fallecido en la contienda, habían emigrado o habían sido depurados por el nuevo régimen político. En el transcurso de la década de los cuarenta, se fueron restableciendo las conexiones con el resto del continente a través de las publicaciones de arquitectura que llegaron

\footnotetext{
${ }^{1}$ Los textos del arquitecto, historiador y crítico Juan Daniel Fullaondo, publicados en la revista Nueva Forma, de la cual era director, resultan imprescindibles para contextualizar históricamente la producción inicial de Fisac. En 1969, esta revista le dedica dos números: FULLAONDO ERRAZU, J. D.: «Miguel Fisac: los años experimentales», Nueva Forma, 39, (abril 1969), pp. 3-4; y FULLAONDO ERRAZU, J. D.: «Miguel Fisac: los años de transición», Nueva Forma, 41, (junio 1969), pp. 3-4.

${ }_{2}$ Entre las numerosas obras que tratan la arquitectura de Fisac, destacamos las siguientes, ya que aluden a la influencia de Japón en su obra: MORALES SARO, M. C.: Miguel Fisac (Un camino en la renovación de la arquitectura española contemporánea, 1942-1972), Universidad de Oviedo, 1974, tesis doctoral publicada en MORALES SARO, M. C.: La arquitectura de Miguel Fisac. Ciudad Real, Colegio de Arquitectos de Ciudad Real, 1979; CÁNOVAS ALCARAZ, A. (editor): Fisac. Medalla de Oro de la Arquitectura 1994. Madrid, Ministerio de Fomento y Consejo Superior de los Colegios de Arquitectos de España, 1997; ARQUES SOLER, F.: La forma y el ornamento en la obra arquitectónica. El Centro de Estudios Hidrográficos de Miguel Fisac. Un para qué, un cómo y un no sé qué (tesis doctoral inédita), Universidad Politécnica de Madrid, 2003; NAVARRO SEGURA, M. I.: «Miguel Fisac: 2003», Basa, 27, (2004), pp. 5-39; DE RODA LAMSFUS, P.: Miguel Fisac. Apuntes y viajes. Madrid, Editorial Scriptum, 2007; y SÁNCHEZ LAMPREAVE, R. (editor): Miguel Fisac: Premio Nacional de Arquitectura 2002, Madrid, Ministerio de Vivienda, 2009.

${ }^{3}$ FERNÁNDEZ ALBA, A.: La crisis de la arquitectura española (1939-1972). Madrid, Cuadernos para el diálogo, 1972, págs. 31-34.
} 




Fig. 2. Miguel Fisac: Edificio Central del C.S.I.C., Madrid, 1943. Foto: Fundación Miguel Fisac.

de nuevo, y, además, muchos arquitectos pudieron viajar al extranjero para conocer de primera mano las corrientes internacionales.

La arquitectura española de posguerra estuvo condicionada por las directrices marcadas por la dictadura de Francisco Franco, que promovía como imagen del régimen una arquitectura nacional, tradicional y monumental, que tenía como modelo la arquitectura de los Austrias y tomaba el Monasterio de El Escorial, obra de Juan de Herrera, como símbolo del Imperio Español. Este hecho imposibilitó la evolución de la incipiente arquitectura moderna que había surgido a finales de la década de los años veinte ${ }^{4}$.

Durante la carrera, Fisac recibió una cierta formación académica con la copia de modelos del natural y de ornamentos arquitectónicos clásicos, así que en sus primeras obras, siguiendo la tendencia predominante en la época en países como Italia o Alemania, se refugió en modelos clasicistas heredados a través del Renacimiento como estrategia para cumplir con los requisitos de monumentalidad e imagen que los encargos del momento requerían. Buen ejemplo de ello es el Edificio Central del Consejo Superior de Investigaciones Científicas (1943) en la Colina de los Chopos de Madrid (fig. 2). Sin embargo, era consciente de que ese no era el camino para hacer una arquitectura de su tiempo, ya que se imponía una mera apariencia exterior a los edificios desvinculada de los problemas reales ${ }^{5}$.

${ }^{4}$ URRUTIA NÚÑEZ, Á.: Arquitectura española. Siglo XX. Madrid, Cátedra, 1997, págs. 353-356.

5 «Pero estas exterioridades de lo clásico no pueden perdurar si se quiere hacer una arquitectura de hoy, porque son demasiado profundos los cambios materiales y espirituales que han ocurrido en nuestro tiempo para que esto suceda». FISAC SERNA, M.: «Lo clásico y lo español», Revista Nacional de Arquitectura, 78, (1948), págs. 197-198. 

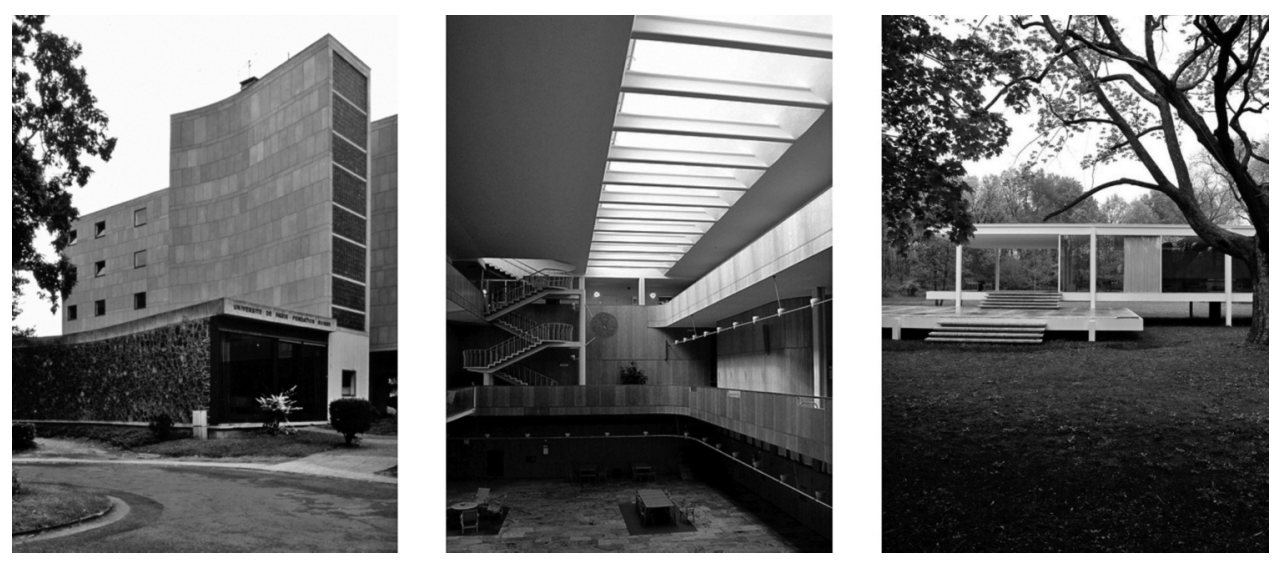

Fig. 3. Le Corbusier: Pabellón Suizo, París (Francia), 1931-1933 (izquierda); Erik Gunnar Asplund: Ayuntamiento de Gotemburgo, (Suecia), 1934-1937 (centro); Ludwig Mies van der Rohe: casa Farnsworth, Plano, (Illinois, EE.UU.), 1951 (derecha).

\section{INFLUENCIA DEL MOVIMIENTO MODERNO EN LA ARQUITECTURA DE MIGUEL FISAC}

Por lo tanto, a medida que va ganando experiencia durante la década de los años cuarenta, Fisac comienza una búsqueda de la esencia de la arquitectura y se plantea otras posibilidades, acercándose a la arquitectura moderna, de influencia nórdica, como se aprecia en edificios de finales de esa década: Biblioteca de la Fundación Hispano-alemana Goerres (1947), Instituto Nacional de Óptica Daza de Valdés (1948), etc. Consecuentemente, realiza una evaluación de los postulados del Movimiento Moderno, a pesar de que desde su época de estudiante se percibía que esa corriente estaba en crisis ${ }^{6}$.

El año 1949 representa un punto de inflexión en la trayectoria de Fisac porque realiza un viaje por Europa donde visita, entre otras obras, el Pabellón Suizo de Le Corbusier (1887-1965) en la Cuidad Universitaria de París (construido entre 19311933), que había visto en revistas y que le resultaba atrayente (fig. 3 izquierda). Sin embargo, quedó decepcionado por esa arquitectura, ya que el Movimiento Moderno le pareció un estilo más, pura estética; aunque también opinaba que, en su momento, tuvo el mérito de proponer el avance de la arquitectura mediante una gran labor propagandística ${ }^{7}$. Como consecuencia, rechazó de plano la arquitectura funcional y racionalista de Le Corbusier:

\footnotetext{
${ }^{6}$ «[...] al salir de la Escuela, andaba yo convencido, como todos los arquitectos, de que el Movimiento Moderno estaba en vía muerta. Sin embargo, aunque más bien poco, tenía el suficiente criterio para juzgar que la mascarada imperialista que se estaba construyendo en España, junto con la otra pseudo-popular en pueblos y aldeas destruidos durante la guerra, tampoco nos conducía a ninguna parte». FISAC SERNA, M.: Carta a mis sobrinos (estudiantes de arquitectura). Madrid, Lampreave y Millán, 2007, pág. 17.

${ }^{7}$ FISAC SERNA, M.: «Estilo moderno», Blanco y Negro, 2358, (13-VII-1957), págs. 91-92.
} 
Con estas apariencias de racionalismo, Le Corbusier no logra encubrir del todo que es un escritor lírico, con unas preocupaciones por la estética difícilmente superadas por arquitectos de otras épocas.

Es innegable, sin embargo, que es el verdadero animador de esta arquitectura y tiene un genio de iniciativa tal que le coloca en primerísimo plano, aunque pierdan fuerzas sus argumentos al llevarlos a las realizaciones prácticas. De una parte, su falta de conocimiento o de cuidado al tratar los materiales, y de otra el agotar hasta unos extremos inadmisibles las consecuencias de sus teorías, hace que sus obras sean mediocres o francamente deplorables... ${ }^{8}$

Por el contrario, en Suecia pudo visitar la ampliación del Ayuntamiento de Gotemburgo construida entre 1934 y 1937, obra de Erik Gunnar Asplund (18851940), quien se convirtió en uno de sus arquitectos de referencia desde entonces (fig. 3 centro):

Me encontré con la obra del entonces recientemente fallecido arquitecto Gunnar Asplund. La actualidad, la honradez constructiva de este gran arquitecto, al que sin haber conocido considero mi maestro, me hizo concebir la esperanza de encontrar un camino nuevo y auténtico a la arquitectura de hoy ${ }^{9}$.

La arquitectura nórdica, incluyendo también la obra del finlandés Alvar Aalto (1898-1976), le interesó por su carácter funcional, pero adaptado a la escala y a las necesidades humanas. También por su construcción con materiales y técnicas locales sin necesidad de una industria muy desarrollada — circunstancias aplicables a la situación española - y por su relación con el paisaje y el entorno donde se inserta ${ }^{10}$.

Otros arquitectos que le resultaron de interés, y de los que posteriormente visitó algún edificio en otros viajes, fueron Ludwig Mies van der Rohe (1886-1969), Frank Lloyd Wright (1867-1959) y Richard Neutra (1892-1970), tal y como analizaremos a continuación.

El primero de ellos, al que Fisac consideraba «tal vez el arquitecto más destacado del siglo $X X{ }^{11}$, fue inicialmente bien valorado por el arquitecto español, quien apreciaba su Pabellón de Barcelona (1929) como una obra de un "estilo lleno de potencialidad creadora» ${ }^{12}$ y «que contenía las mayores sugerencias de realización "13. Asimismo, admiraba a Mies van der Rohe porque había conseguido «dar expresividad plástica arquitectónica al acero laminado, que llevaba más de medio siglo utilizándose como material estructural [...] de for-

\footnotetext{
${ }^{8}$ FISAC SERNA, M.: La arquitectura popular española y su valor ante la del futuro. Madrid, Ateneo de Madrid, 1952, pág. 19.

${ }^{9}$ FISAC SERNA, M.: Panorama español contemporáneo. Madrid, Editorial Cultura Hispánica, 1964, pág. 261.

${ }^{10}$ MORALES SARO, M. C.: Op. Cit., pág. 26.

${ }^{11}$ FISAC SERNA, M.: Carta a mis sobrinos..., pág. 11.

12 Idem, pág. 15.

${ }^{13}$ Ibidem.
} 


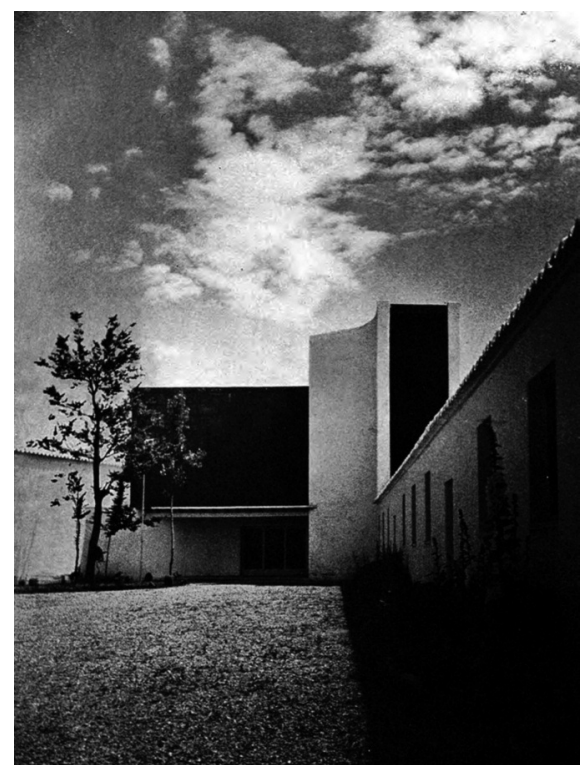

Fig. 4. Miguel Fisac: Instituto Laboral, Daimiel, 1952.

ma rutinaria y sin gracia» ${ }^{14}$. Sin embargo, la visita a la casa Farnsworth (1951), cerca de Chicago (EE.UU.), en 1955 le decepcionó profundamente (fig. 3 derecha). Primeramente, por su mala ubicación en un lugar pantanoso, pero, sobre todo, porque percibió que la arquitectura de Mies es pura estética mantenida inmutable en el tiempo a pesar de las circunstancias tan cambiantes de la sociedad, y además sin ninguna preocupación por el lugar donde se asienta, llegando en ocasiones a proponer la misma solución formal para proyectos situados en entornos totalmente diferentes ${ }^{15}$.

Por otro lado, Fisac se acercará al concepto de la arquitectura orgánica de Wright hacia 1949, gracias a las investigaciones arquitectónicas, funcionales y formales, que lleva a cabo cuando intenta definir en esa época su propio método para proyectar; así como al reencontrarse con la arquitectura popular de su tierra cuando se le presenta la ocasión de construir varios proyectos en La Mancha. De entre ellos, destaca el Instituto Laboral de Daimiel (1952), en el que aplica por primera vez sus nuevas ideas (fig. 4). Realiza una arquitectura en la que tiene muy en cuenta las funciones a las que responde, pero que está compuesta de forma menos rígida que sus anteriores obras y buscando la relación con el entorno natural donde se ubica. Respecto a este edificio, Fisac manifestaba:

${ }^{14}$ Idem, pág. 45.

${ }^{15}$ Véase FISAC SERNA, M.: «Las dos traiciones», ABC, (22-IX-1972), pág. 19. 
Yo no tenía noticia de la arquitectura orgánica y escasísima de la americana y aquello que hice, sin ninguna referencia exterior, a mí me pareció que se parecía algo en su disposición a los órganos de los seres vivientes y, solo para mi uso personal por la mala eufonía del neologismo, le llamé arquitectura de mondongo; pues este es el nombre que dan en mi tierra al aparato digestivo de los rumiantes, y esta era la analogía viviente que encontraba más afín con mi solución arquitectónica ${ }^{16}$.

En cuanto a Neutra, Fisac mantuvo una amistad con el arquitecto austriaco desde que este visitó por primera vez nuestro país en 1954 con motivo de una conferencia en el Instituto Técnico de la Construcción y el Cemento. Con él compartía su actitud de adaptación de la arquitectura al ser humano, para cubrir sus necesidades tanto biológicas como psicológicas; su preocupación por el entorno donde se sitúan sus edificios, ya que establece relaciones entre su arquitectura y la naturaleza; así como su refinado sentido del paisaje.

\section{INFLUENCIA DE LA TRADICIÓN EN LA ARQUITECTURA DE MIGUEL FISAC}

Como ya se ha comentado, durante los primeros años de la década de los cincuenta, Fisac construye varios edificios en su tierra volviendo a encontrarse de esta manera con la tradición de la arquitectura popular española. La enseñanza que se puede extraer de la arquitectura popular fue un asunto que los arquitectos españoles tomaron como punto de partida para reflexionar sobre la renovación de la arquitectura nacional desde las primeras décadas del siglo XX. Este tema se mantuvo vigente en el debate arquitectónico español incluso con la irrupción de las corrientes internacionales modernas, como por ejemplo, las ideas de Le Corbusier en los años veinte.

Las aportaciones de Fisac a esta cuestión se presentan esencialmente en dos pequeñas monografías: La arquitectura popular española y su valor ante la del futuro ${ }^{17}$ y Arquitectura popular manchega ${ }^{18}$, que recogen sendas ponencias del arquitecto; la primera, una conferencia en el Ateneo de Madrid en 1951; y la segunda, su discurso de ingreso en el Instituto de Estudios Manchegos en 1985.

En ellas, Fisac resalta el sentido práctico de la arquitectura popular, en la que las necesidades programáticas y estructurales se combinan de tal manera que es imposible disociarlas. Al ser una arquitectura construida con los mínimos elementos para hacerla más económica, cada componente responde directamente y de forma sencilla a los requisitos planteados. Se genera de esta manera una arqui-

${ }^{16}$ FISAC SERNA, M.: Carta a mis sobrinos..., págs. 29 y 31.

${ }^{17}$ FISAC SERNA, M.: Op. Cit.

${ }^{18}$ FISAC SERNA, M.: Arquitectura popular manchega. Ciudad Real, Colegio Oficial de Arquitectos de Ciudad Real, 2005, publicado por primera vez como separata de Cuadernos de Estudios Manchegos, 16, (1985). 

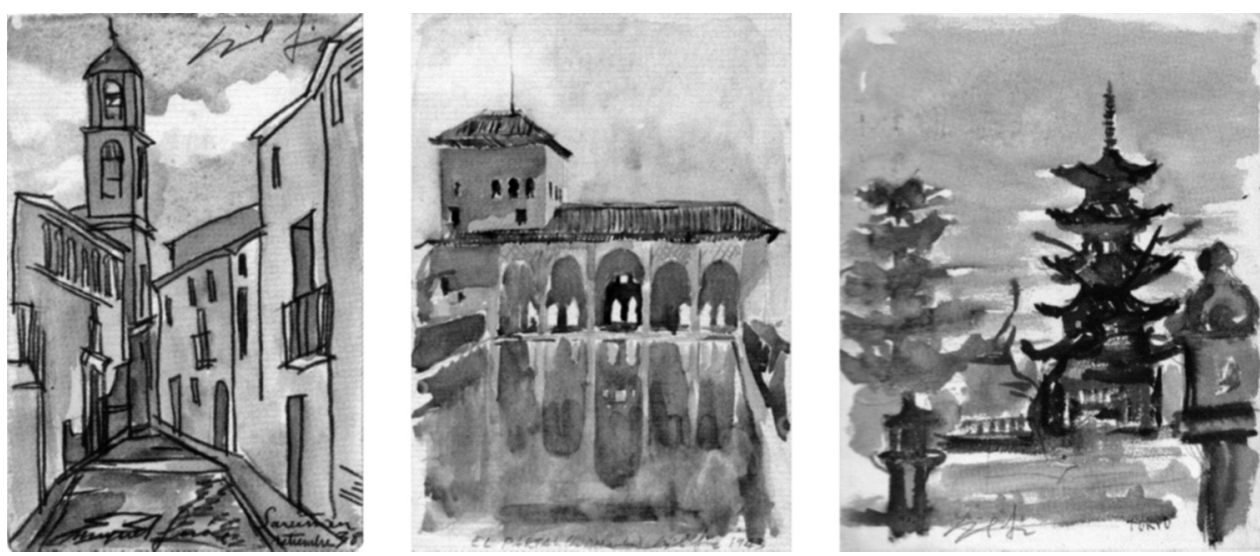

Fig. 5. Acuarelas de Miguel Fisac: Saviñán, 1938 (izquierda); El Partal (La Alhambra), 1943 (centro); Tokyo, 1953 (derecha).

tectura intemporal, cuya bondad reside en su utilidad. Por otra parte, los elementos de estas edificaciones se relacionan de forma orgánica entre sí, con cierto carácter espontáneo; mientras que tomada en conjunto, este tipo de arquitectura se encuentra vinculada con su entorno e integrada en el paisaje. Ante la arquitectura popular, Fisac muestra una actitud en línea con los criterios de arquitectos anteriores como, por ejemplo, Teodoro de Anasagasti (1880-1938), quien durante su magisterio en la Escuela de Arquitectura de Madrid organizaba excursiones con sus alumnos para tomar apuntes de arquitectura popular ${ }^{19}$; hábito este que Fisac también cultivará en sus viajes durante toda su vida (fig. 5).

Como consecuencia, el arquitecto manchego toma estos valores intemporales de la arquitectura popular española como referente para la arquitectura contemporánea y del futuro porque, en su opinión, pueden servir para «aprender de ella honradez, verdadero funcionalismo y, sobre todo, amor al paisaje, que es el primer escalón para empezar a quererse los hombres, para que puedan convivir»20.

Pero no es esta la única tradición que atraerá a Fisac durante su carrera. También extraerá interesantes conclusiones tanto de la arquitectura islámica, principalmente de La Alhambra de Granada, como de la arquitectura tradicional japonesa, la cual se tratará en un apartado aparte.

En cuanto a su relación con el palacio andalusí, hay que señalar que Miguel Fisac fue uno de los firmantes del conocido Manifiesto de La Alhambra de $1952^{21}$, fruto de

\footnotetext{
${ }^{19}$ VÁZQUEZ ASTORGA, M.: José Borobio. Su aportación a la arquitectura moderna. Zaragoza, Delegación del Gobierno en Aragón, 2007, pág. 62.

${ }^{20}$ FISAC SERNA, M.: La arquitectura popular española..., pág. 27.

${ }^{21}$ CHUECA GOITIA, F. et alii: Manifiesto de La Alhambra. Granada, Fundación Rodríguez-Acosta y Delegación en Granada del Colegio Oficial de Arquitectos de Andalucía Oriental, 1993.
} 
las Sesiones Críticas de Arquitectura que la Revista Nacional de Arquitectura promovía desde 1950. Una de estas sesiones dio pie a que se organizara una visita al monumento para extraer de La Alhambra valores arquitectónicos que favorecieran la renovación de la arquitectura española, encargando a Fernando Chueca Goitia la tarea de recopilar y redactar las conclusiones del debate. Según el análisis del propio manifiesto, la arquitectura española estaba en crisis desde el desastre de 1898, cuando se produjo el derrumbamiento del Imperio colonial español, y el intento de recuperación de esa tradición imperial tras la Guerra Civil era en esos momentos insostenible.

Efectivamente, ya en 1949 se había puesto de manifiesto el cambio de tendencia en el panorama nacional en la $V$ Asamblea Nacional de Arquitectos celebrada en Barcelona ${ }^{22}$, el cual se materializó con la construcción de la Casa Sindical (1949-1951) de Francisco de Asís Cabrero y Rafael Aburto²3, y se desarrolló de forma imparable en la década de los cincuenta ${ }^{24}$.

Volviendo a La Alhambra, para Fisac, las enseñanzas que extrajo de ella supusieron una aportación más para la definición de su propia arquitectura:

En resumen La Alhambra me ha enseñado: [...] Que la arquitectura es espacio, no volumen. [...] Que el espacio puede humanizarse limitándolo geométricamente. [...] Que tiene grandísima importancia el tratamiento que se dé a las superficies limitantes de ese espacio. [...] Que La Alhambra nos recuerda que tenemos cinco sentidos. [...] Que tiene una importancia capital la forma de articular los diferentes espacios de un edificio. [...] Que la vertebración de todo el conjunto responde a una concepción trascendente de la vida humana ${ }^{25}$.

\section{INFLUENCIA DE ORIENTE EN LA ARQUITECTURA OCCIDENTAL}

Oriente, concretamente la arquitectura tradicional japonesa, fue una referencia fundamental en la obra de Fisac. Si rastreamos el comienzo de esta atracción, veremos que resulta ser muy temprana: «Me empezó a interesar Oriente en la época de cuando estaba estudiando: yo creía que Japón tenía cosas. Y además veía que la gente que hacía la arquitectura entonces se inspiraba mucho en lo japonés» ${ }^{26}$.

Ciertamente, las referencias orientales se pueden encontrar abundantemente en la cultura occidental a finales del siglo XIX en pleno auge del Japonismo. Esta corriente afectó a todas las manifestaciones artísticas, favoreciendo la renovación

\footnotetext{
${ }^{22}$ FLORES LÓPEZ, C.: Arquitectura Española Contemporánea, I: 1880-1950. Madrid, Aguilar, 1989, págs. 249-265.

${ }^{23}$ FULLAONDO ERRAZU, J. D.: «Miguel Fisac: los años experimentales»...

${ }^{24}$ FERNÁNDEZ ALBA, A.: Op. Cit., págs. 35-41.

${ }^{25}$ FISAC SERNA, M.: «Lo que he aprendido en La Alhambra», conferencia pronunciada en el Colegio de Arquitectos de Granada el 21-IV-1994, publicada en CÁNOVAS ALCARAZ, A.: Op. Cit., págs. 10-11. ${ }^{26}$ DE RODA LAMSFUS, P.: Op. Cit., pág. 294.

${ }^{27}$ ALMAZÁN TOMÁS, D.: «La seducción de Oriente: de la Chinoiserie al Japonismo», Artigrama, 18, (2003), págs. 83-106.
} 


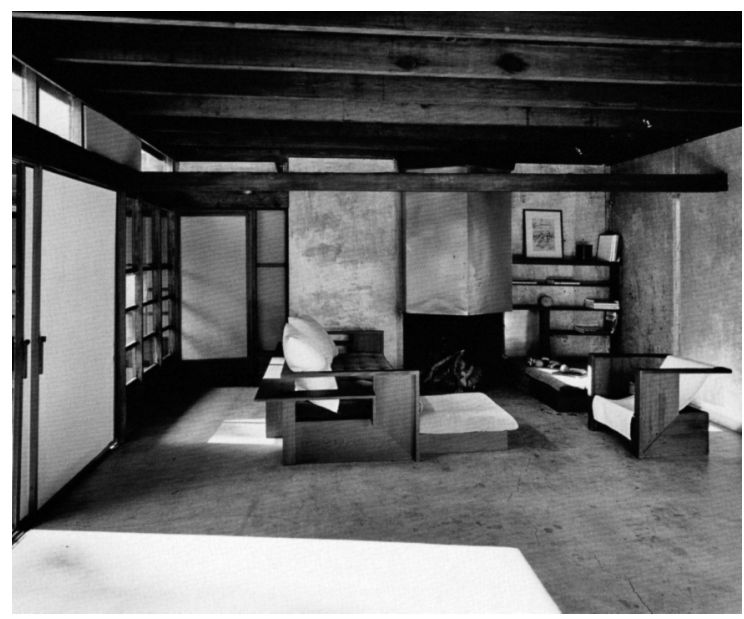

Fig. 6. Rudolf Michael Schindler: casa en Kings Road, Los Ángeles (California, EE.UU.), 1921-1922.

de las artes occidentales ${ }^{27}$. Por supuesto, también influyó a los arquitectos. Primeramente, a los vinculados con el Art Nouveau, y, posteriormente, a numerosas y prominentes figuras decisivas en la generación del Movimiento Moderno.

Entre todas ellas, el arquitecto sobre el que más se ha investigado en relación con su interés por el arte japonés y la influencia en su obra es, sin duda, Frank Lloyd Wright ${ }^{28}$. Son numerosos los artículos y monografías que de una forma más o menos profunda apuntan su vínculo con Japón y su condición de coleccionista de obras de arte orientales desde fecha tan temprana como 1900, antes incluso de que Wright visitara el país por primera vez en 1905 o residiera en él (entre 1917 y 1922) con el fin de construir allí varios proyectos ${ }^{29}$.

De esta forma, se han señalado numerosas similitudes entre detalles de sus edificios y diversos elementos de la arquitectura japonesa, además de tener un concepto espacial parecido. Por otra parte, tal y como declaró en varias ocasiones a lo largo de su vida, la importancia que Wright otorga al espacio en su obra proviene de las ideas del filósofo chino Lao $\mathrm{zi}^{30}$ : «La realidad del edificio no consiste en las paredes y el techo, sino en el espacio interior en el que se vive ${ }^{31}$.

Tomando como punto de partida el trabajo de Wright, se observa que la inspiración en la arquitectura japonesa de la arquitectura moderna estadounidense es palpable en otros casos. Uno de los más claros es la obra inicial del arquitecto aus-

\footnotetext{
${ }^{28}$ Para un análisis de las influencias japonesas en la obra de Frank Lloyd Wright, véase NUTE, K.: Frank Lloyd Wright and Japan. Londres, Chapman \& Hall, 1993.

${ }^{29}$ Un estudio completo de la colección de Wright y sus actividades como marchante de arte oriental puede encontrarse en MEECH, J.: Frank Lloyd Wright and the Art of Japan. Nueva York, Harry N. Abrams, 2001.

${ }^{30}$ VAN DE VEN, C.: El espacio en la arquitectura. Madrid, Cátedra, 1981, pág. 309. Para una visión más completa de los conceptos espaciales de Lao zi, véase el capítulo primero de esta obra.

${ }^{31}$ El texto original en inglés es: "The reality of the building does not consist in roof and walls but in the space within to be lived in». WRIGHT, F. L.: A Testament. Nueva York, Horizon Press, 1957, pág. 155.
} 


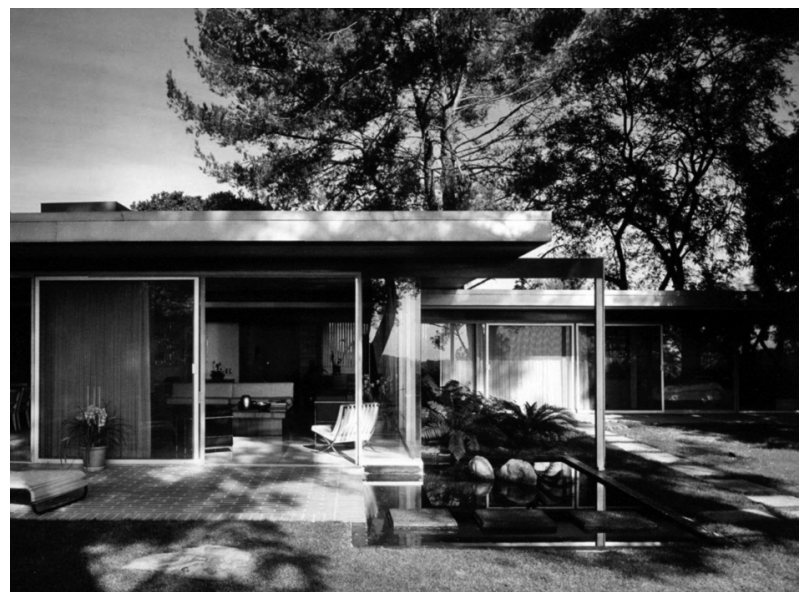

Fig. 7. Richard Neutra: casa Singleton, Los Ángeles (California, EE.UU.), 1959.

triaco Rudolf Michael Schindler ${ }^{32}$ (1887-1953), quien, después de trabajar para Wright, construyó numerosas viviendas en Estados Unidos. Entre ellas destaca la adaptación moderna de la arquitectura tradicional japonesa que proyectó para su propia casa en Kings Road, Los Ángeles, construida en 1922 (fig. 6).

Otro arquitecto austriaco relacionado con conceptos arquitectónicos nipones es Richard Neutra, quien también trabajó en Estados Unidos con Wright y Schindler. En la obra de Neutra se puede percibir tanto un afán de integración de los edificios en la naturaleza, como un gusto por las distribuciones asimétricas similares a las que se dan en las viviendas tradicionales japonesas (fig. 7). Neutra pudo apreciar la arquitectura y los jardines orientales en una estancia que realizó en Japón, donde pronunció varias conferencias en el verano de $1930^{33}$. Tras su viaje por Oriente publicó varios artículos en la revista alemana Die Form, medio de expresión del Deutscher Werkbund ${ }^{34}$.

Algunas de las causas de este interés por Japón en los Estados Unidos son, por una parte, la labor de difusión de Ernest Francisco Fenollosa (1853-1908) y de su discípulo Kakuzo Okakura (1862-1913), que realizaron desde el Museo de Bellas Artes de Boston a partir de 1890. Por otra parte, cabe citar el éxito de publicaciones como Japanese Homes and Their Surrondings (1886) del arquitecto Edward Sylvester Morse (1838-1925) —que estuvo en Japón entre 1877 y 1883 estudiado de primera mano las viviendas tradicionales japonesas-. También, las obras de Okakura, entre las que se encuentra El libro del té (1906), que es la más

${ }^{32}$ STEELE, J.: Schindler. Colonia, Taschen, 2005, pág. 11.

${ }^{33}$ MAC LAMPRECHT, B.: Neutra. Complete Works. Colonia, Taschen, 2010, pág. 38.

${ }^{34}$ Comunicación presentada por Barbara Mac Lamprecht, recogida en KIM, H. S.: «East Asian Influence on Modern Architecture in Europe, 1918-1939. Session report of the 60th Annual Meeting of Society of Architectural Historians, Pittsburgh, Pennsylvania, 9-15 April 2007», Journal of Architectural History, v. 16, n. 53, (2007), págs. 173-181. 
Hacia la esencia de la arquitectura: el papel de Oriente en los años experimentales...

difundida y que recoge el concepto taoísta de Lao zi citado anteriormente: «solo en el vacío [...] reside lo verdaderamente esencial. La realidad de una habitación, por ejemplo, no se encuentra en el techo o las paredes, sino en el espacio que estos elementos dejan vacío» ${ }^{35}$.

Entre los arquitectos europeos interesados en la cultura nipona podemos destacar al alemán Bruno Taut (1880-1938), el cual residió en Japón en el periodo 1933-1936, publicó varios escritos sobre la arquitectura y el modo de vida japonesas, y construyó varias viviendas en aquel país ${ }^{36}$.

Menos conocido es el interés por la cultura oriental, especialmente china, de otros destacados arquitectos alemanes como son Hans Scharoun (1893-1972) y Hugo Häring ${ }^{37}$ (1882-1958) —quien colaboró con Bruno Taut dentro de la actividad de la asociación berlinesa de arquitectos progresistas Der Ring, fundada en $1926^{38}$ —. Estos dos arquitectos llevaron a cabo varios encuentros para discutir sobre arquitectura china entre 1941 y 1942, por lo que esta influencia (de la arquitectura y de la planificación de las antiguas capitales de China) está presente en algunos de sus trabajos de arquitectura orgánica y urbanismo.

Por lo tanto, se puede comprobar que entre los arquitectos alemanes hubo un interés por Oriente, al que no fue ajeno el maestro de la arquitectura Ludwig Mies van der Rohe. Así se deduce de su colección de libros de filosofía china, entre los que se encuentran las obras de Confucio y de Lao zi, y también por su conocimiento de la filosofía budista zen a través de los escritos de Daisetsu Teitaro Suzuki ${ }^{39}$.

Otro foco interesante en cuanto a la influencia oriental en la arquitectura moderna se halla en el norte de Europa. En los últimos años se ha puesto de manifiesto la inspiración japonesa en la arquitectura del finlandés Alvar Aalto en ciertos detalles de su Villa Mairea ${ }^{40}$ (construida entre 1938-1939), la cual estuvo muy influida por las descripciones del libro Das Japanische Wohnhaus (1935) del arquitecto japonés Tetsuro Yoshida (1894-1956). Esta obra tuvo gran resonancia entre los arquitectos europeos gracias a la labor de establecimiento de relaciones que su autor mantuvo durante la gira que realizó por Europa durante un año (entre 1931 y

\footnotetext{
${ }^{35}$ OKAKURA, K.: El libro del té. Madrid, Miraguano, 2001, págs. 52 y 54.

${ }^{36}$ Véase, entre otras publicaciones, TAUT, B.: La casa y la vida japonesas. Barcelona, Fundación Caja de Arquitectos, 2007.

${ }^{37}$ Véase BLUNDELL JONES, P.: «The Lure of the Orient: Sharoun and Häring's East-West connections», Architectural Research Quarterly, v. 12, n. 1, (2008), págs. 29-42; y HÄRING, H.: «Conversation with Chen Kuan Lee about roof profiles (1947)», Architectural Research Quarterly, v. 12, n. 1, (2008), págs. 26-28.

38 GARCÍA ROIG, J. M.: Tres arquitectos alemanes. Bruno Taut, Hugo Häring, Martin Wagner. Valladolid, Secretariado de Publicaciones e Intercambio Editorial, Universidad de Valladolid, 2004, págs. 1314.

${ }^{39}$ Para un completo análisis de las referencias orientales en la obra del arquitecto alemán, véase BLASER, W.: West meets East, Mies van der Rohe. Basilea, Birkhäuser, 2001.

${ }^{40} \mathrm{KIM}, \mathrm{H}$. S.: «A Study on the Influence of Japanese Tokonoma on Aalto's Art Display Concept in Villa Mairea (1937-39)», Journal of Architectural History, v. 15, n. 47, (2006), págs. 43-57.
} 
1932) con base en Berlín, teniendo a Häring como enlace con los arquitectos alemanes ${ }^{41}$.

Por otra parte, el arquitecto sueco Erik Gunnar Asplund (1885-1940), quien conoció personalmente a Yoshida, también valoró muy positivamente los recursos que la arquitectura nipona ofrecía. Así lo manifestó en su discurso de nombramiento de profesor en el Real Instituto de Tecnología de Estocolmo en 1931, haciendo referencia a la flexibilidad de la casa japonesa como modelo de adaptación de la vivienda occidental a los requerimientos de sus usuarios ${ }^{42}$.

Consecuentemente, tal como manifestaba Fisac, en el momento en el que estudiaba la carrera de arquitectura - es decir, en la década de los años treinta y primeros años cuarenta - la influencia oriental en Occidente había sido digerida, entre otras referencias, y formaba parte de alguna forma de la arquitectura del Movimiento Moderno. Estas corrientes internacionales volvieron a penetrar en el país gracias a las revistas extranjeras que empezaron a llegar en la segunda mitad de los años cuarenta. En principio, se tomaron de manera dogmática, descontextualizadas y desvinculadas del debate arquitectónico que las había generado, para posteriormente, en la década de los cincuenta, abrazar el discurso moderno de manera generalizada y más rigurosamente ${ }^{43}$.

Esto no significa que no existan influencias orientales en la arquitectura española. Tal y como se ha explicado, este influjo está latente en la obra de los maestros extranjeros. Simplemente la historiografía nacional las ha obviado para centrarse en otros temas ${ }^{44}$. En relación a esto, el caso de Fisac nos muestra que España no fue tan impermeable a las referencias orientales como se puede pensar a tenor de lo publicado sobre la historia de la arquitectura moderna española.

\section{INFLUENCIAS ORIENTALES EN EL PENSAMIENTO DE MIGUEL FISAC}

Como ya se ha señalado anteriormente, Fisac lleva a cabo en los años cuarenta una búsqueda personal de la esencia de la arquitectura que le oriente frente al complicado panorama arquitectónico español de posguerra: «[...] una frase atribuida a Lao-Tsé, "Cuatro paredes y un techo no son arquitectura, sino el es-

\footnotetext{
${ }^{41} \mathrm{KIM}, \mathrm{H}$. S.: «Tetsur Yoshida (1894-1956) and architectural interchange between East and West», Architectural Research Quarterly, v. 12, n. 1, (2008), págs. 43-57.

${ }^{42}$ ASPLUND, E. G.: «Our Architectural Conception of Space», Architectural Research Quarterly, v. 5, n. 2, (2001), págs. 151-160.

${ }^{43}$ FERNÁNDEZ ALBA, A.: Op. Cit., págs. 27-31.

${ }^{44}$ En concreto, en la ruptura de la introspección arquitectónica nacional, superando el eclecticismo y los regionalismos de principios de siglo mediante la entrada de la arquitectura moderna europea a partir de la generación de 1925; la actividad del GATEPAC (Grupo de Artistas y Técnicos Españoles para el Progreso de la Arquitectura Contemporánea) a partir de 1930; las repercusiones de la Guerra Civil en el panorama arquitectónico nacional; y la recuperación de la modernidad hacia 1950. Véase FLORES LÓPEZ, C.: Op. Cit.
} 
pacio que queda dentro", y que leí no sé si en algo referido a Wright, me puso en la pista del concepto espacial y esencial de la arquitectura» ${ }^{45}$. En efecto, ya se ha citado esta idea en relación con los conceptos espaciales de Frank Lloyd Wright. Ese pensamiento del legendario filósofo chino Lao zij6 (o Lao-Tsé, como escribe Fisac), que aparece escrito en el siglo III a.C. en una recopilación de textos que recogen su doctrina, es considerado como la manifestación más antigua de la idea de espacio arquitectónico de la que se tiene constancia ${ }^{47}$.

A principios de los años cincuenta, tras una década de ejercicio profesional, Fisac tenía ya claro cuál era su concepto de arquitectura y había desarrollado su método personal para proyectar ${ }^{48}$. Es en ese momento cuando entra en contacto con Oriente:

Con todos estos criterios en mi poder, me lancé entonces a buscar en la Historia de la Arquitectura los ejemplos de espacialidad arquitectónica en los que pudiera aprender y encontré uno, para mí bastante exótico y casi desconocido, en la arquitectura japonesa, no monumental sino popular ${ }^{49}$.

En 1953, tras construir el Colegio Apostólico de Arcas Reales para los Padres Dominicos en Valladolid, le surge la posibilidad de impartir un ciclo de conferencias en la Facultad de Arquitectura de la Universidad de Santo Tomás en Manila (Filipinas), invitado por el Provincial de los Dominicos como experto de arquitectura religiosa; oportunidad esta que aprovecha para visitar Japón por primera vez. Por otra parte, realizó otra estancia en ese país en 1955, incluida en su viaje alrededor del mundo 50 (fig. 8).

En estas estancias entró en contacto con la civilización oriental y pudo apreciar la gran tradición cultural de Japón, lo cual expresará en sus artículos posteriores. Uno de los temas que trata es el del paisaje, cuyos elementos, tal y como ya había visto en la pintura japonesa, tienen también en la realidad «un decorativismo salvaje y extraño ${ }^{51}$, ya que han sido moldeados según la estética nipona para acentuar su belleza y formar parte del conjunto como se hace en las artes plásticas. Percibe de esta manera el concepto oriental de paisaje, el cual se remonta a la

\footnotetext{
${ }^{45}$ FISAC SERNA, M.: Carta a mis sobrinos..., pág. 27.

46 «Treinta radios convergen en el cubo de una rueda, / y es de su vacío, / del que depende la utilidad del carro. / Modelando el barro se hacen vasijas, / y es de su vacío, / del que depende la utilidad de las vasijas de barro. / Se horadan puertas y ventanas, / y es de su vacío, / del que depende la utilidad de la casa. / El ser procura ganancia, / El no-ser procura utilidad». LAO ZI: Lao zi: el libro del Tao. Madrid, Ediciones Alfaguara, 1983, pág. 111.

${ }^{47}$ VAN DE VEN, C.: Op. Cit., pág. 309.

${ }^{48}$ Una explicación sobre su proceso mental de proyecto se encuentra en FISAC SERNA, M.: «Itinerario profesional», en ARQUES SOLER, F.: Miguel Fisac. Madrid, Ediciones Pronaos, 1996, págs. 35-39.

${ }^{49}$ FISAC SERNA, M.: Carta a mis sobrinos..., pág. 33.

${ }^{50}$ Se recogen artículos de cada una de estas etapas del viaje en la revista La Actualidad Española: «Atenas» (203, 24-XI-1955), «Jerusalén» (205, 8-XII-1955), «Calcuta» (207, 24-XII-1955), «Bangkok» (209, 5-I-1956), «Manila» (211, 19-I-1956), «Tokio» (213, 2-II-1956), «San Francisco, Los Ángeles, Hollywood» (215, 16-II-1956), «Chicago» (217, 1-III-1956) y «Nueva York» (219, 15-III-1956).

${ }^{51}$ FISAC SERNA, M.: «Tokio», La Actualidad Española, 213, (2-II-1956), págs. 11-13.
} 


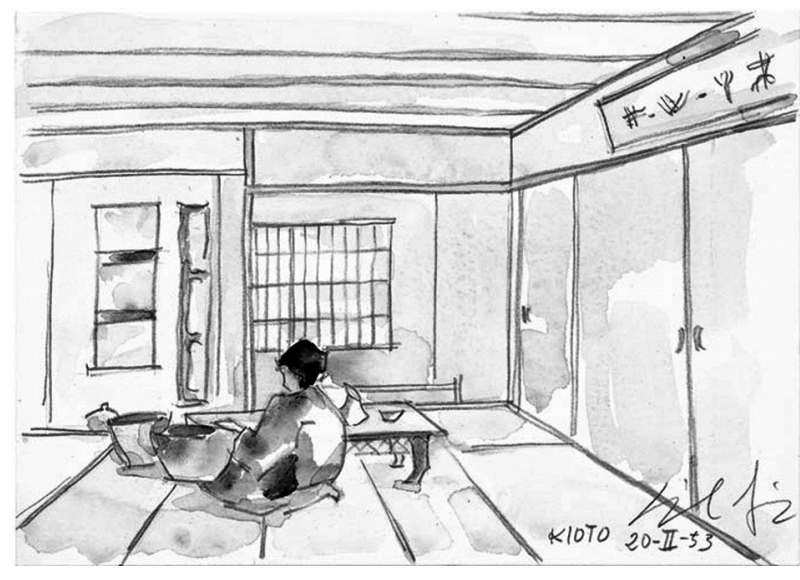

Fig. 8. Acuarela de Miguel Fisac: Kioto, 1953.

China del siglo V, cuando el poeta Xie Lingyun escribió: «El sentimiento, por el gusto, genera la belleza", proyectando su sensibilidad sobre el entorno natural y generando de esta manera la primera idea de paisaje ${ }^{52}$. Según esto, no hay inconveniente en modificar el entorno -obviando el trabajo que esto implica- para adaptarlo al gusto, y crear así el paisaje deseado. Esta noción se extenderá posteriormente a Japón; mientras que en Occidente, no será hasta el siglo XIV cuando aparezca con Petrarca un pensamiento parecido, que no se desarrollará prácticamente hasta el siglo XVI.

Fisac señala también que «toda esa rara belleza que flota en lo japonés procede de una refinadísima cultura estética, a la que nunca se ha llegado en Occidente " ${ }^{53}$, lo cual da muestra de la fascinación que despertó en el arquitecto español esa inclinación hacia lo bello que se manifiesta en los más insignificantes detalles de la vida cotidiana japonesa.

Por ejemplo, es en el arte de colocar las flores (ikebana) donde se perciben tanto los principios estéticos como morales de la idiosincrasia nipona. Por una parte, se crea una dialéctica entre las tensiones generadas con la colocación de cada elemento y el equilibrio del todo; y, por otra, este sistema de composición tiene resonancias filosóficas porque sirve de ejemplo del dominio de la propia voluntad en favor del equilibrio del conjunto. Fisac apunta también que, a diferencia del arte occidental que se hace para durar, los japoneses se deleitan con la creación de arreglos que solo duran unos días, siendo este arte efímero y cambiante como la vida misma. «Eso forma parte de una serie de talentos que afinan el gusto ${ }^{54}$, comentaba Fisac en relación a la enseñanza que extrajo del arte floral.

\footnotetext{
${ }^{52}$ BERQUE, A.: El pensamiento paisajero. Madrid, Editorial Biblioteca Nueva, 2009, págs. 74-79.

${ }^{53}$ FISAC SERNA, M.: «Tokio»...

${ }^{54}$ DE RODA LAMSFUS, P.: Op. Cit., pág. 294.
} 
También se refiere en términos parecidos a la ceremonia del té (chanoyu), a la que tuvo ocasión de asistir, que con su depuración estética es a la vez de una gran belleza sensorial y un momento de reflexión e introspección: «La ceremonia del té es un ritual para meditar, para estar con paz..., por no decir unos ejercicios espirituales» ${ }^{55}$.

El origen de esta sensibilidad lo pone Fisac en la educación japonesa -tema al que, de hecho, dedicó un artícul ${ }^{56}$ - la cual fomenta la formación afectiva además de la racional. Es decir, que en Japón se valoran actividades que cultivan la moral y la estética formando la personalidad de manera más completa porque engloban la parte intelectual, pero también la afectiva. No como en Occidente, donde prima la formación intelectual «y que nos lleva a una supercivilización de salvajes: sin sentido moral, sin sentido cívico y sin sentido estético ${ }^{57}$. Este concepto tan negativo que tiene Fisac de la civilización occidental se puede percibir en sus numerosos artículos y obras que abordan problemas de las ciudades como el tráfico, el progreso técnico, la convivencia humana, la relación con el entorno o la pérdida del patrimonio arquitectónico. Ciertamente, el urbanismo fue la gran batalla de Fisac durante toda su vida, lo cual le llevó a escribir en 1969 La molécula urbana, un libro donde recoge sus ideas urbanísticas y propone un nuevo modelo de ciudad ideal para el futuro ${ }^{58}$.

Mención aparte requiere la casa japonesa, a la que dedicó varios escritos y que fue uno de sus mayores referentes, confirmándole «el concepto espacial de la arquitectura llevado a su más radical realización ${ }^{59}$ y reafirmándole «en el sentido antropomórfico de la arquitectura adintelada» ${ }^{60}$.

Estas son ideas que se manifiestan de forma clara en su casa en el Cerro del Aire, proyectada en 1956. La vivienda se encuentra a las afueras de Madrid, en un lugar elevado desde el que se domina el paisaje. Se sitúa en el fondo de la parcela, en la parte más alta de solar, accediendo a ella a través de un jardín por un camino sinuoso. Consta de una sola planta que se ha organizado en sucesivas ampliaciones alrededor de un patio interior, con vegetación y un pequeño estanque, que recuerda a los jardines interiores de las casas japonesas (tsuboniwa). La parcela ajardinada también tiene este mismo carácter oriental por su vegetación aparentemente silvestre. Por otra parte, en las fachadas predomina la horizontalidad de la losa de hormigón armado de cubierta y la casa se distribuye de forma asimétrica, remitiendo a la obra de la primera época de Mies y a la de Neutra, pero también a la composición arquitectónica nipona (figs. 9-11).

\footnotetext{
${ }^{55}$ Idem, pág. 296.

${ }^{56}$ FISAC SERNA, M.: «La lección de enseñanza en Japón», ABC, (26-VII-1953), págs. 8-9.

57 Ibidem.

${ }^{58}$ FISAC SERNA, M.: La molécula urbana. Madrid, Ediciones y Publicaciones Españolas, 1969.

59 FISAC SERNA, M.: Carta a mis sobrinos..., pág. 33.

60 Ibidem.
} 

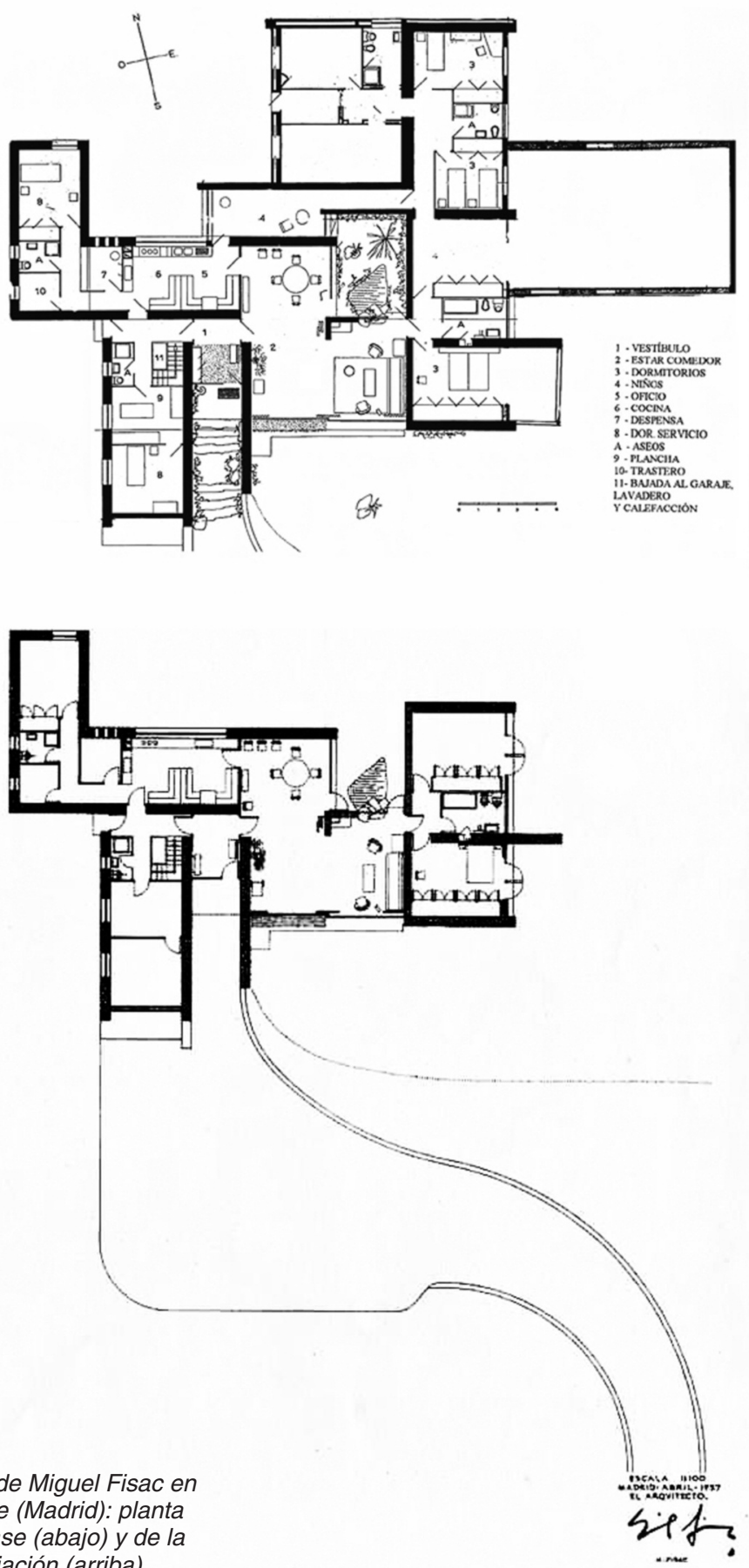

Fig. 9. Vivienda de Miguel Fisac en de la primera fase (abajo) y de la cuarta ampliación (arriba). 


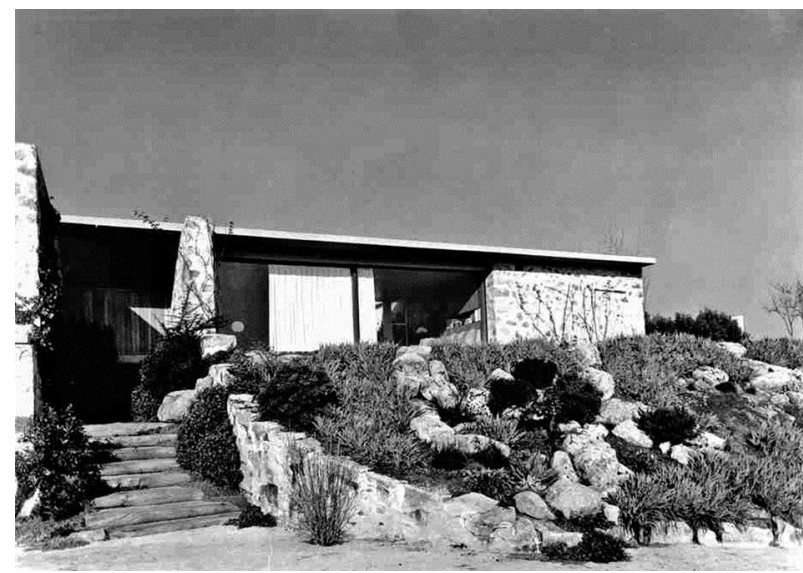

Fig. 10. Vivienda de Miguel Fisac en el Cerro del Aire (Madrid): acceso. Foto: Fundación Miguel Fisac.

Para Fisac la vivienda japonesa es un ejemplo de flexibilidad y sencillez, ya que su espacio se adapta a las múltiples funciones con los mínimos recursos y puede resultar aplicable a los requerimientos de la vivienda moderna61:

La casa tradicional japonesa es, a mi manera de ver, la que de forma más clara y desnuda cumple el clásico programa de la vida de una familia [...].

En esencia, el programa de la casa japonesa es la creación permanente de dos superficies que humanizan el espacio habitado entre ellas.

La de arriba protege de las inclemencias atmosféricas; de la lluvia [...].

La plataforma inferior separa la vida familiar del contacto directo con la tierra y es a la vez una forma clara de resolver el problema de las humedades.

Todo lo demás en la casa japonesa es mudable. Las paredes exteriores son puertas correderas de madera opaca o estructuras ligerísimas, también de madera, con papel pergamino pegado o, ahora, cristales.

El interior se compartimenta con unos tabiques de papel más fuerte, en general ornamentado con dibujos de paisajes, flores o pájaros.

Cuando se entra en un espacio de casa japonesa, siempre está vacío. Posteriormente la función crea el órgano y surgen, traídos por reverenciales mujeres, tazones de té, mesas de laca para comer, «jibachis» de porcelana con lumbre para calentarse, colchones para dormir, etc. Ya sé que todo esto supone mucho trabajo, inaceptable para la trabajosa vida de hoy, pero encierra la enseñanza del esquema puro del programa de la vivienda ${ }^{62}$.

61 «[...] como el problema de la vivienda nos exige un profundo replanteo de todas sus cualidades y características, con vistas a lo que debe ser el programa de la vivienda del futuro, ella [la casa popular japonesa] nos puede servir de pauta para buscar el resultado que buscamos». FISAC SERNA, M.: «Ciudad, espacio para vivir», Los Domingos de ABC, (20-II-1972), pp. 10-11.

${ }^{6}$ FISAC SERNA, M.: Mi estética es mi ética. Ciudad Real, Museo de Ciudad Real, 1982, págs. 28-29, artículo publicado originalmente en FISAC SERNA, M.: «Programa de vivienda mínima», Diario Pueblo, (27-II-1964). 


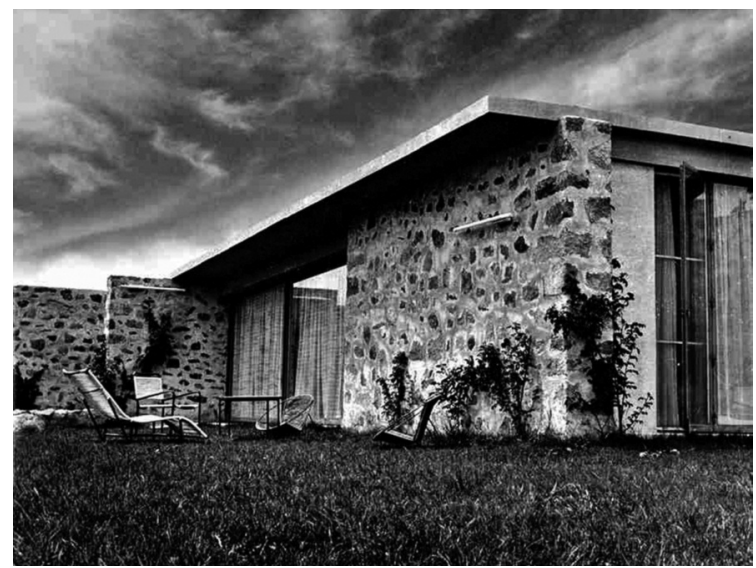

Fig. 11. Vivienda de Miguel Fisac en el Cerro del Aire (Madrid): fachada sur. Foto: Fundación Miguel Fisac.

Pero Fisac no fue el único que consideraba este modelo. Como hemos dicho, también Asplund (uno de sus mayores referentes) se había manifestado anteriormente en esta misma línea.

De esta manera, el interior de la casa en el Cerro del Aire es amplio, diáfano, se expande a través del patio ajardinado, por un lado; y, por el otro, hacia el paisaje. Fisac utilizó materiales naturales, dando importancia a sus texturas, que deja a la vista: muros portantes de mampostería de granito, muros de fábrica de ladrillo, revestimientos de madera de roble en techo y paredes, etc. A pesar de su sencillez y austeridad, consigue también una gran calidez, inspirada, por una parte, en lo popular; por otra, en la arquitectura nórdica de Aalto, la cual tomó los acabados naturales en los interiores por influencia nipona (fig. 12).

Además, la casa se fue amueblando con los prototipos de muebles que el arquitecto creó durante su carrera profesional. Fisac diseña su casa haciéndola económica, práctica de utilizar y ampliable según las necesidades futuras, pero, a pesar de su contención, también usando materiales que transmiten una sensibilidad estética depurada. Esto proviene de la vivienda japonesa, la cual el arquitecto manchego valora por «su sencillez programática, su claridad constructiva y dispositiva, su elasticidad y transformabilidad de espacios, la profunda humanización de sus dimensiones y la pureza de sus resultados plásticos» ${ }^{63}$. Asimismo, por su vinculación con la naturaleza. La relación con el entorno natural se produce mediante la ubicación de la vivienda en un jardín que remite al paraíso original perdido, donde no hacía falta la arquitectura, ya que la naturaleza no era hostil para el ser humano ${ }^{64}$. Así lo interpreta

${ }^{63}$ FISAC SERNA, M.: «Ciudad, espacio para vivir»...

${ }^{64}$ FISAC SERNA, M.: Mi estética es mi ética. Ciudad Real, Museo de Ciudad Real, 1982, págs. 26-27, artículo publicado originalmente en FISAC SERNA, M.: «Funciones humanas de la vivienda», Diario Pueblo, (21-II-1964). 


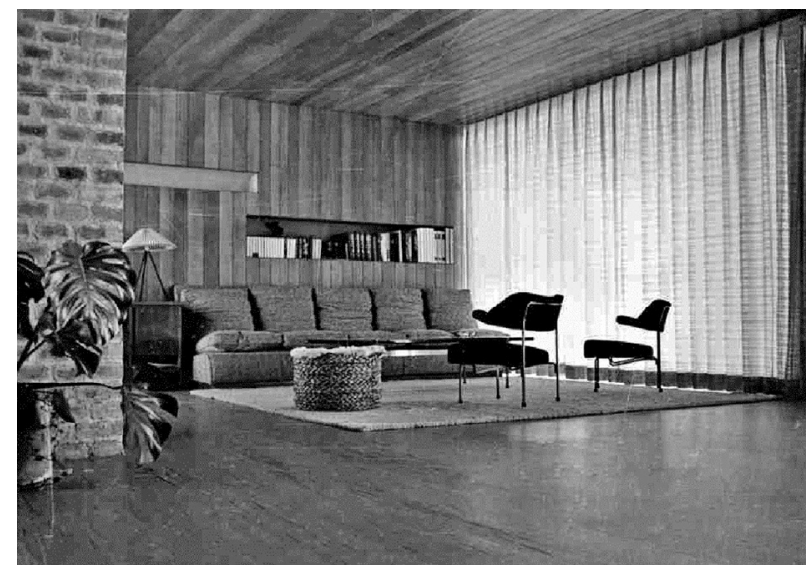

Fig. 12. Vivienda de Miguel Fisac en el Cerro del Aire (Madrid): interior. Foto: Fundación Miguel Fisac.

Fisac a través de su pensamiento profundamente cristiano. Por lo tanto, la jardinería era un tema que no descuidaba en sus edificios y que se manifestó en sus proyectos desde época temprana.

Consecuentemente, en esta casa, como había hecho Wright en su arquitectura, la distinción entre interior y exterior se diluye: el salón se vuelca hacia el exterior mediante grandes superficies acristaladas, que se pueden abrir comunicando el espacio interior con el jardín, el cual se eleva hasta la cota de la vivienda. Muchos de los rasgos descritos en esta casa, se encuentran de nuevo en otros de sus viviendas posteriores: Luca de Tena (1960), Fisac (1961), Barrera (1962), Tejada (1967), Casacuberta (1968), etc.

Finalmente, señalaremos que, al igual que la casa popular japonesa, la vivienda en el Cerro del Aire cumple perfectamente con la definición que Fisac daba de lo que era arquitectura: «un trozo de aire humanizado» ${ }^{65}$, es decir, la acotación de un espacio para acondicionarlo a las necesidades humanas tanto físicas (de protección frente a las condiciones climáticas, haciéndolo un lugar habitable) como también espirituales (rodeando al ser humano de belleza).

Todas estas referencias orientales ayudaron, sin duda, a Fisac a perfilar su concepto arquitectónico por la cantidad de veces que hace alusión a ellas en sus escritos y por sus declaraciones:

La casa japonesa es punto y aparte. Confieso paladinamente que ni la arquitectura clásica y renacentista, ni los grandes maestros contemporáneos me han enseñado más que ella. En la casa japonesa la arquitectura está en su sitio: como subsidiaria de la Naturaleza. Para ayudarla y suplirla cuando no hay más remedio ${ }^{66}$.

\footnotetext{
65 Ibidem.

${ }^{66}$ FISAC SERNA, M.: «Tokio»...
} 
El contacto con la arquitectura nipona favoreció el cambio que se produjo en la obra de Fisac en la década de los cincuenta, cuando comienza a proyectar sus edificios con una mayor flexibilidad espacial mediante la disposición de espacios comunicados entre sí. También, busca la sinceridad arquitectónica en la estructura, en el papel de los materiales, así como en la relación entre la función y la forma de todos los elementos que componen la construcción. Por otra parte, se produce una pérdida de monumentalidad en sus edificios en favor de la creación de conjuntos orgánicos adaptados a la escala humana donde prima la horizontalidad. Además, hay que señalar un afán de integración en el paisaje de su arquitectura, desornamentada, de líneas depuradas, que tiende hacia lo esencial ${ }^{67}$.

Los años experimentales, en los que Fisac recorre diferentes caminos en la búsqueda de sus propias herramientas para proyectar, concluyen a finales de los años cincuenta. A partir de 1959, cambia de registro y comienza una nueva etapa caracterizada por una unidad de estilo que deriva de sus investigaciones sobre la prefabricación en la arquitectura y el hormigón armado como material de construcción, lo cual le lleva a plantear nuevos sistemas constructivos como sus conocidas vigas-hueso y sus encofrados flexibles.

\section{CONCLUSIONES}

En el presente texto se ha ofrecido una panorámica del desarrollo intelectual y arquitectónico de Miguel Fisac durante las décadas de los años cuarenta y cincuenta. Desde el inicio de su carrera profesional en plena posguerra, evoluciona, tal y como hemos analizado, tomando referencias de diferentes corrientes arquitectónicas: arquitectura clásica, neoempirismo nórdico, organicismo, arquitectura popular, tradición islámica, etc. De igual manera, se ha estudiado el influjo que otros arquitectos extranjeros, especialmente los representativos del Movimiento Moderno, ejercieron sobre su pensamiento.

También, se han presentado las considerables influencias orientales, especialmente japonesas, que existen en la arquitectura moderna occidental. El influjo de la arquitectura nipona que se produjo desde finales del siglo XIX y que estuvo presente durante la gestación del Movimiento Moderno, fue digerido finalmente por esta corriente internacional. Fisac no desconocía este hecho, lo cual despertó en él la curiosidad por viajar a Japón, según deducimos de sus palabras. De esta forma, el arquitecto español visitó aquel país en varias ocasiones, tal y como hemos señalado, y tuvo interés en su cultura, de la cual extrajo ciertas enseñanzas que aplicó posteriormente en su arquitectura. Por lo tanto, retomó de alguna manera esas referencias orientales, las estudió de primera mano en sus viajes y las aplicó en su producción de una forma mucho más auténtica, volviendo a su origen, y po-

${ }^{67}$ Véase MORALES SARO, M. C.: Op. Cit., págs. 61-63. 
tenciando en su obra un espíritu mucho más fiel a la estética japonesa de lo que la arquitectura moderna occidental estaba realizando en la década de los cincuenta.

Por otra parte, queremos hacer notar que los arquitectos con los que Fisac se identificó y que le resultaron más interesantes fueron, precisamente, aquellos que se inspiraron en la arquitectura oriental. Con ellos coincide en otorgar una gran importancia al espacio, de forma que se expanda de manera orgánica; en los criterios de humanización de la arquitectura mediante la adecuación a la escala humana y por el uso de materiales naturales; así como en la necesidad de integración de los edificios con el entorno, valorando muy cuidadosamente la relación con la naturaleza y el respeto hacia el paisaje. Por lo tanto, consideramos que este arquitecto debe encuadrarse dentro de esa corriente de renovadores de la arquitectura occidental que tomaron Oriente como referencia.

Para muchos artistas y arquitectos de finales del siglo XIX y principios del XX, las influencias orientales posibilitaron la exploración de nuevas vías y facilitaron la renovación de las artes occidentales. Para el arquitecto manchego, esos mismos modelos le sirvieron como ayuda para concretar una nueva visión de su arquitectura, incorporando criterios de flexibilidad espacial y composición mediante el juego de tensiones y equilibrio, además de ser un ejemplo de un profundo sentido estético conseguido mediante la sencillez y con una gran economía de medios. Así se ha comprobado a través del estudio de los artículos que Fisac escribió posteriormente como consecuencia de sus viajes a Japón y con el análisis de su vivienda en el Cerro del Aire, que se ha puesto como ejemplo.

Oriente para Fisac supuso la culminación de su búsqueda arquitectónica personal porque descubrió, a través de las reflexiones de Lao zi y entrando en contacto con la jardinería y la casa japonesas, la esencia tanto del paisaje como de la arquitectura. 
